\title{
Correction: Determination of Kamlet-Taft parameters for selected solvate ionic liquids
}

Cite this: Phys. Chem. Chem. Phys., 2016, 18, 19975

DOI: $10.1039 / c 6 c p 90177 h$

\section{Daniel J. Eyckens, ${ }^{\text {ab }}$ Baris Demir, ${ }^{\text {a }}$ Tiffany R. Walsh, ${ }^{a}$ Tom Welton $^{c}$ and} Luke C. Henderson ${ }^{\star a b}$

Correction for 'Determination of Kamlet-Taft parameters for selected solvate ionic liquids' by Daniel J. Eyckens et al., Phys. Chem. Chem. Phys., 2016, 18, 13153-13157.

www.rsc.org/pccp

The authors would like to make the following corrections to their article:

(a) In the abstract and conclusion, $\beta$ values for the G3TFSI and G4TFSI should be amended to $\beta=0.31$ and $\beta=0.28$, respectively.

(b) In Table 1, the $\beta$ values and footnote ' $b$ ' should be amended as shown below:

Table 1 Polarity (EN) and Kamlet-Taft paramters for G3TFSI, G4TFSI, G3, G4, and [bmim]TFSI

\begin{tabular}{lllrr}
\hline Solvent & $E_{\mathrm{T}}^{\mathrm{N}}$ & $\alpha^{a}$ & $\beta^{b}$ & $\pi^{*}$ \\
\hline G3TFSI & 1.03 & 1.32 & 0.31 & 0.28 \\
G4TFSI & 1.03 & 1.35 & 0.71 & 0.90 \\
G3 Glyme & 0.30 & 0.01 & 0.72 & 0.65 \\
G4 Glyme & 0.28 & 0.05 & 0.22 & 0.67 \\
[bmim][TFSI] $^{\text {[bmim][TFSI] }}{ }^{c}$ & 0.59 & 0.59 & 0.24 & 0.96 \\
\end{tabular}

${ }^{a}$ Normalised against methanol. ${ }^{b}$ Normalised against HMPA. ${ }^{c}$ Previously published polarity and Kamlet-Taft values for this IL, ref. 26 and 31.

The Royal Society of Chemistry apologises for these errors and any consequent inconvenience to authors and readers.

\footnotetext{
${ }^{a}$ Institute for Frontier Materials, Deakin University, Waurn Ponds Campus, Geelong, Victoria, 3216, Australia. E-mail: luke.henderson@deakin.edu.au

${ }^{b}$ Strategic Research Centre for Chemistry and Biotechnology, Deakin University, Waurn Ponds Campus, Geelong, Victoria, 3216, Australia

${ }^{c}$ Department of Chemistry, Imperial College London, South Kensington, London, SW7 2AZ, UK
} 